Post-refereed, preprint version of the text published at European Journal of Health Law 2013(3)289-94. Link to the publisher's website: http://www.brill.com/european-journal-health-law

Sirpa Soini

\title{
FINLAND ON A ROAD TOWARDS A MODERN LEGAL BIOBANKING INFRASTRUCTURE
}

\begin{abstract}
Finland has enacted a Biobank Act that will come into force on September 1, 2013. Finland is regarded for many reasons as a highly successful environment for medical research that uses population samples and data. One of the rationales behind the new legislation was to solve problems due to the overly strict informed consent doctrine hindering access to old samples and data and asking for multi-purpose consents. Yet, consent remains the primary justification to use biobank samples and data. The Biobank Act allows asking for consent for several unspecified future research purposes. The guiding principles of the Biobank Act are promotion of trust, equal access to data and samples, protection of privacy, acceleration of innovation activities, and bringing biobank activities under public scrutiny. To the author's knowledge, this is the first "all purpose" Biobank Act in Europe that applies to all biobanks in a country.
\end{abstract}

\section{Main features of the new law}

Finland has enacted a Biobank Act (Act 688/2012) ${ }^{1}$ that will come into force on September 1, 2013. At the same time provisions of the Tissue Act that regulates secondary use of human biological samples will become amended. One of the rationales for the Biobank Act was to solve problems due to the overly strict informed consent doctrine (i.e., the limited scope of the consent) and enable secondary uses of old samples and data.

To my knowledge, this is the first "all purpose" Biobank Act in Europe applied to all biobanks, whether clinical or research based, public or private, and irrespectively of the identifiable or anonymous nature of data and samples. Clinical trials and other onetime research protocols will remain as stipulated by the Medical Research Act (488/1999). The novelty is that the Biobank Act allows asking for a consent for several unspecified future research purposes in which biobank samples and data can be used.

The guiding principles of the Biobank Act are a promotion of trust, equal access to data and samples, protection of privacy, acceleration of innovation activities, and bringing biobank activities under public scrutiny. Translational research, personalised medicine, the benefits of public-private collaboration and the optimal use of resources for the ultimate goal of improved delivery of health care services are explicitly mentioned in the travaux preparatoires (Governmental proposal 86/2011).

A biobank owns the samples and data in its possession, unless otherwise agreed explicitly in the material transfer agreement. A biobank is obliged to publish

\footnotetext{
" The author, LL.M Sirpa Soini is a senior legal adviser at the National Institute for Health and Welfare (THL), Helsinki, Finland; legal adviser for the Finnish national biobank coordination, www.bbmri.fi; and a member of the Governmental Expert Steering Group for the Implementation of the Biobank Act, appointed by the Ministry of Health and Social Affairs.

${ }^{1}$ www.finlex.fi Finnish official page for government acts, bills, case-law etc. Some acts have been translated into other languages, but the Biobank Act is not yet one of them.
} 
information about its samples and their use for biobank research, and the results thereof. The idea is that data will accumulate in the biobank for the benefit of other researchers, thus avoiding repetition of certain analyses, and bringing new findings onto a common platform.

A biobank can collect, store, analyse and share human biological samples and related geno- and phenotype data, obtained directly from the research subject or through accessing clinical data and health-based registries, or by various analyses. Use of samples and data are subject to strict coding requirements, unless otherwise consented to.

\section{Finland's strengths in biobank research}

Finland is regarded for many reasons as a highly successful environment for medical research that uses population samples and data. First, the Finns have a relatively isolated gene pool which is an advantage for genetic and molecular studies. ${ }^{2}$ The unique personal identity numbers, introduced in 1964, and early computerised registers combined with patient records and biological samples provide a rich and versatile source for public health research and longitudinal population studies. Further, church records contain information about births, marriages and deaths from as early as the $17^{\text {th }}$ century, so population trends can be tracked. ${ }^{3}$ Like our Scandinavian neighbours, we are still ranked high regarding trust in biomedical science and in the state. ${ }^{4}$ All these advantages contribute to successful, high quality research. Indeed, many ongoing epidemiological studies date back several decades, and their value as a research resource continues to accumulate. ${ }^{5}$ The key question is how to optimally use these resources, while at the same time maintaining people's trust.

\section{Establishment of a biobank is subject to ethical review}

As of September 1, 2013 a legal entity can establish a biobank in Finland. The legal entity can be a public or private operator: a hospital, a research institute, a pharmaceutical, biotechnological or other company, a foundation, inter alia, as long as its economic and functional operations as well as legal and scientific preconditions are adequate. A single researcher cannot be a founder, but he or she can transfer valuable collections beneficial to future research purposes into a biobank, and also access the biobanks for own research purposes.

An application for an ethical review to establish a biobank shall be submitted to the National Medical Research Ethics Committee (TUKIJA). The application shall contain substantial amounts of information regarding the owner, funder, repository, registries, field of activities, access criteria and principles, consent procedures, applied model documents, and operating plans with a description of the organisation, responsibilities,

\footnotetext{
2 See L. Peltonen, A. Jalanko, T. Varilo, "Molecular genetics of the Finnish disease heritage”. Human Molecular Genetics, 1999; 8 (10): 1913-1923.

3 See M. Gissler and J. Haukka, "Finnish health and social welfare registers in epidemiological research". Norsk Epidemiologi 2004; 14 (1): 113-120.

${ }^{4}$ G. Gaskell et al., "Europeans and biotechnology in 2010. Winds of change?” European Commission, 2010 (EUR 24537).

${ }^{5}$ See www.nationalbiobanks.fi These pages contain information on Finnish epidemiological and clinical study collections and their availability for research.
} 
and breadth and level of activities. TUKIJA has sixty days in which to give its statement, conditional on no further information being needed.

\section{Commencement of biobank operations requires registration}

Biobanks will be supervised by the National Supervisory Authority for Health and Welfare (Valvira). Before starting its operations, a biobank must file a register application to Valvira to be registered in the National Biobank Register. The application shall include documents showing the legal status; statement of TUKIJA; nomination of "the person in charge of the biobank" and his or her training and experience; accounts for risk management and quality control; organisation chart, personnel, capacities and responsibilities; person register notifications; and list of standard operating procedures.

Valvira guides the biobank activities and has competence to intervene in the event it finds deficits in a biobank's activities. It can make inspections and give orders (e.g., obligate the biobank to give access to its samples and data) and injunctions.

\section{Consent is the primary justification to use biobank samples and data}

The use of samples and data for biobank research shall be primarily based on a person's informed consent. The biobank-specific consent can be seen as consent for inclusion of samples and data in several research projects in a specified biobank. Yet, there are still some who consider this kind of consent to be too broad and thus illegitimate. However, under the Biobank Act the person shall be adequately informed about the owner of the biobank and about the biobank which stores the samples and data, as these may be different bodies. The registered field of activities of the biobank serves as a limitation for the use of its samples and data. Further, the person to be recruited shall be informed of the general nature of biobanking and potential risks, the purposes of collection and storage, the voluntariness, the right to cancel or limit the consent at any time. The person may also give consent that his personal data may be disclosed, or be linked to register data.

One of the concerns is how to keep the consent valid and fresh in the course of time, and how to better empower individuals to govern the use of their samples and data. One proposed solution is to integrate the management of one's consent into the National Archive of Health Information (KanTa), which is currently being developed to serve health care, pharmacies and citizens. The services, once fully operational, will include electronic prescription, a Pharmaceutical Database, a My Health Information, and a Patient Records Archive. Ideally, a person could then manage and access all his or her health data through KanTa, with biobank consents included. This might be a step towards "the dynamic consent", as suggested by the European Commission Expert Group in 2012.6

Clinical and research samples that have been collected before the Biobank Act comes into force (i.e., September 1, 2013), can be transferred to a biobank as a result of a specific notification procedure and subsequent opt-out principle. The precondition is that a regional ethics committee approves the planned transfer. In addition, the

\footnotetext{
${ }^{6}$ European Commission, "Biobanks for Europe. A challenge for governance". Report of the Expert group on Dealing with Ethical and Regulatory Challenges of International Biobank Research. European Commission, 2012 (EUR 25302).
} 
persons involved shall be contacted with the information about the transfer into a biobank, with the idea that if he or she does not object, the samples and related data will be transferred to a biobank. Should the samples be old, numerous or the persons hard to reach with reasonable efforts, the notification procedure can instead occur through public media. A consent is not required for the transfer, even though preferred; therefore I call this procedure an opt-out mechanism.

The Tissue Act still holds for some exemptions regarding the use of cadaveric, diagnostic or therapeutic samples and tissues for secondary medical research purposes and transfer into biobanks under certain criteria. Nevertheless, it is worth noting that research samples and data cannot be used for secondary purposes after the Biobank Act is effective without a new consent. Thus, it is important to assess future needs when making research protocols to incorporate potential needs into the consent procedure. Naturally, the candidate biobank for storage shall exist is such cases, as a consent is always related to some existing biobank and its field of activities.

\section{The premise of equal access to biobank material}

Biobank samples and data can be used for biobank research if the intended use complies with the notified field of research activities for a given biobank and with the consent or other basis, for instance, resulting from the transfer of old samples. In addition, research and handling of samples and data shall follow relevant laws and rules, and the recipient shall have an appropriate professional and scientific competence. A proper link to recipient's tasks is also required.

A party wishing to initiate biobank research has a right to access the biobank's metaregister to find out whether relevant research material is available. Once a party desires to access the samples and data, it shall make a requisition along with a research plan or other necessary specification on the intended use. Depending on the research plan, a research ethics review may also be needed.

The Biobank Act's starting point is that biobanks are shared research resources. Therefore, the provisions state that a biobank can restrict delivery of its samples and data only if justified after considering the following points:

- the intended use is against the biobank's field of activities and other restrictions,

- safeguarding ongoing primary research,

- safeguarding IPR,

- preservation of rare or limited precious collections for very significant purposes,

- safeguarding privacy (small collection, where data exposure is likely),

- reasons related to research ethics.

Same access criteria apply to both the biobank's own and to external research. A biobank must develop a transparent process describing its access policy. These will be explicitly tested in the administrative courts, if necessary.

\section{A register subject's right to receive information}

A register subject, as he or she is called in the Act, can ask whether his or her samples are being stored in a biobank, the grounds for storage, from where related data has been collected and to where disclosed. In addition, a register subject can ask about health-related information resulting from the analyses, in which case he or she shall be 
offered a possibility to know the meaning of such information. This legal obligation assigned to biobanks raises great concerns: is this feasible, in particular, in research laboratories that are not accredited for clinical purposes, and hence, cannot usually give clinically valid results?

\section{Conclusions}

Despite some ambiguities and some outstanding challenges, the political will and support is very strong in guiding Finnish biobanking towards success. It is also obvious that the Biobank Act was long awaited among the Finnish biobank community and thus, all the relevant actors are committed to working together for the common goal. For instance, relevant actors are developing harmonised data standards and systems, consent practices etc., so that the interoperability of biobanks will be a reality from the beginning. The focus of attention is also on joining the European wide biobank research infrastructure, BBMRI-ERIC, a legal entity under EU legislation. ${ }^{7}$

All five university hospitals (Helsinki, Turku, Tampere, Kuopio and Oulu) will create their own clinical biobanks. Time will demonstrate if and how they specialise in some therapeutic areas. In addition, a Finnish Cancer Centre is likely to be established soon. Further, the National Institute for Health and Welfare (THL) will establish a population-based biobank, to which it intends to transfer its major longitudinal cohorts. The development of the inventory is ongoing. The plans of private actors are so far unknown.

The Ministry of Health and Social Affairs has appointed an expert steering group for a period of five years to monitor the implementation and functionality of the Biobank Act, and to suggest amendments to it, when considered necessary.

The momentum to develop a strong comprehensive biobank research infrastructure with good practices remains currently strong, with the clear intention of overcoming hurdles and identifying the needs of all the stakeholders, so that opportunities are not missed.

\footnotetext{
${ }^{7}$ www.bbmri.eu
} 